\title{
Triangulations of the sphere, bitrades and abelian groups
}

\author{
Simon R. Blackburn* and Thomas A. McCourt ${ }^{\dagger}$ \\ June 11, 2021
}

\begin{abstract}
Let $\mathcal{G}$ be a triangulation of the sphere with vertex set $V$, such that the faces of the triangulation are properly coloured black and white. Motivated by applications in the theory of bitrades, Cavenagh and Wanless defined $\mathcal{A}_{W}$ to be the abelian group generated by the set $V$, with relations $r+c+s=0$ for all white triangles with vertices $r, c$ and $s$. The group $\mathcal{A}_{B}$ can be defined similarly, using black triangles.

The paper shows that $\mathcal{A}_{W}$ and $\mathcal{A}_{B}$ are isomorphic, thus establishing the truth of a well-known conjecture of Cavenagh and Wanless. Connections are made between the structure of $\mathcal{A}_{W}$ and the theory of asymmetric Laplacians of finite directed graphs, and weaker results for orientable surfaces of higher genus are given. The relevance of the group $\mathcal{A}_{W}$ to the understanding of the embeddings of a partial latin square in an abelian group is also explained.
\end{abstract}

\section{Introduction}

Let $\mathcal{G}$ be a triangulation of the sphere, where each triangle is coloured either black or white, and where no two triangles of the same colour share a common edge, i.e. $\mathcal{G}$ is a (properly) face 2 -coloured triangulation. Let $W$ be the set of

*Department of Mathematics, Royal Holloway, University of London, Egham, Surrey TW20 0EX.

${ }^{\dagger}$ Heilbronn Institute for Mathematical Research, School of Mathematics, University of Bristol, University Walk, Bristol BS1 1TW. 
white triangles, and let $B$ be the set of black triangles. We assume that $\mathcal{G}$ is finite (indeed, all objects in this paper are finite unless indicated otherwise).

Heawood [16] showed that there is a (proper) vertex 3-colouring of $\mathcal{G}$ : see for example the proof of Theorem 4.11 in Wilson [20, Page 38]. Let $R, C$ and $S$ be the colour classes, so $V=R \cup C \cup S$ in this colouring and every triangle contains exactly one vertex from each of $R, C$ and $S$.

Define an abelian group $\mathcal{A}_{W}$ using the set of white triangles as follows: $\mathcal{A}_{W}$ is the abelian group with generating set $V$, subject to the relations $\{r+c+s=0:\{r, c, s\} \in W\}$. Define $\mathcal{A}_{B}$ from the set of black triangles in $\mathcal{G}$ in a similar way: $\mathcal{A}_{B}$ has the same generating set $V$ as $\mathcal{A}_{W}$, but relations $\{r+c+s=0:\{r, c, s\} \in B\}$.

We prove the following theorem:

Theorem 1. For any triangulation $\mathcal{G}$ as above, $\mathcal{A}_{W} \cong \mathcal{A}_{B}$.

This establishes the truth of a well-known conjecture by Cavenagh and Wanless [6, Conjecture 1]; see the 17th edition of the Kourovka Notebook [17, Problem 17.35] or the list of problems from the 21st British Combinatorial Conference [3, Problem 524, BCC22.21]. The motivation for this problem came from a conjecture on latin bitrades due to Cavenagh and Drápal [7]; we provide more details in Section 2, (Incidentally, the origins of this problem in the theory of latin trades explains our choice of notation for the colour classes of our triangulation: the triangle $\{r, c, s\}$ corresponds to an entry of symbol $s$ in row $r$ and column $c$ of a partial latin square.)

Let $G$ be the graph embedded in the sphere to form $\mathcal{G}$ and let $V$ be the vertex set of $G$. For an edge $e$ of $G$, we write $\psi(e)$ for the set of its two end vertices. Every triangle is uniquely determined by its colour together with the set of three distinct incident vertices. Note that it is possible for there to be two triangles of different colours with the same associated triple of vertices; the simplest example is when we have two faces that are identified with opposite hemispheres, with a triangle on the equator between them, and Figure 1 gives a more complicated example. This example also shows that an edge is not necessarily determined by its end vertices (so $G$ is not necessarily simple); moreover, the rotation at a vertex (in other words, the boundary of the union of all triangles containing a vertex) is not necessarily a simple cycle. Triangulations in which the rotation at every vertex is a simple cycle are sometimes known as piecewise-linear triangulations. Since all triangulations arising from latin bitrades are piecewise linear, our Theorem 1 is, in fact, a little more general than the application to latin bitrades needs. 


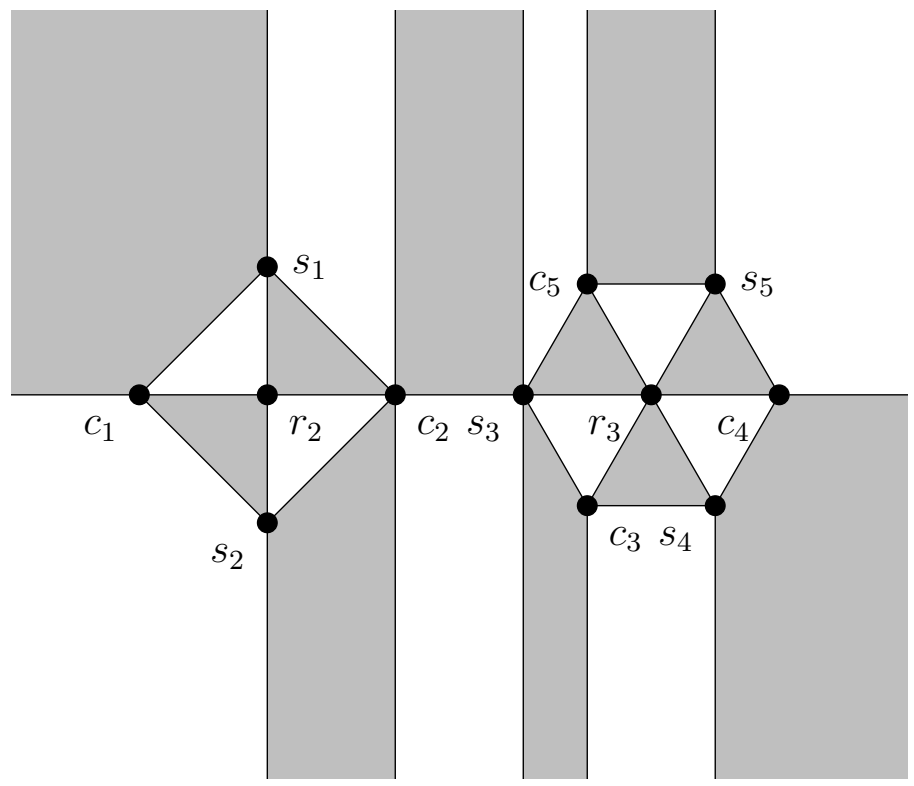

Figure 1: A triangulation. One node $\left(r_{1}\right)$ has been placed at infinity.

The structure of the rest of this paper is as follows. In Section 2, we discuss how our problem arises in the study of spherical latin bitrades. The results and terminology in this section are not needed to understand the main results of this paper, but motivate these results and are needed to understand some of our comments on possible future directions at the end of this paper. In Section 3, we prove a preliminary lemma on connected planar bipartite graphs. Theorem 1 is proved in Section 4. We establish more information about the structure of the group $\mathcal{A}_{W}$ in Section 5, connecting this group with the theory of asymmetric Laplacians on directed graphs. In particular, we use this theory to provide examples of groups $\mathcal{A}_{W}$ of maximal possible rank, and of exponential order. Finally, in Section 6, we give examples to show that Theorem 1 cannot hold for general surfaces, and comment on the form of $\mathcal{A}_{W}$ and $\mathcal{A}_{B}$ in this more general case. We also provide a selection of open problems in the area. 


\section{Background and motivation}

Let $G$ be a graph (not necessarily simple) with $v$ vertices. If the edges of $G$ can be partitioned into isomorphic copies of $K_{3}$, then such a partition is called a partial triple system of order $v$; moreover if the maximum number of edges in $G$ that have the same end points is $\lambda$, then the partial triple system is said to have index $\lambda$, we write $\operatorname{PTS}(v, \lambda)$.

Now consider a partial triple system, $\operatorname{PTS}(v, \lambda)$ say, that is vertex 3colourable and let $n$ be the size of the largest colour class. The system, together with a vertex 3-colouring, is called a partial transveral design of block size three and index $\lambda$; we write $\operatorname{PTD}_{\lambda}(3, n)$. Moreover, if each pair of vertices from distinct colour classes occur in precisely $\lambda$ triples (hence, $v \equiv 0(\bmod 3)$ and the graph $G$ partitioned into copies of $K_{3}$ is the $\lambda$-fold complete tripartite graph $\left.\lambda K_{v / 3, v / 3, v / 3}\right)$, then it is said to be a transveral design of block size three and index $\lambda$. Finally, if $\lambda=1$ we say that the (partial) transversal design is called a (partial) latin square. The order of a partial latin square is the size of the largest colour class (in other words, $n$ ). See Part III of [8] for a survey of results on latin squares.

Let $P$ be a partial latin square, of order $n$, with vertex colouring represented by a partition of the vertices into the sets $R$ (the rows), $C$ (the columns) and $S$ (the symbols). Note that $R, C$ and $S$ are by definition pairwise disjoint. We can relabel the vertices in $R$ (respectively, $C$ and $S$ ) as $r_{i}$ (respectively, $c_{j}$ and $s_{k}$ ), indexed by a set of size $|R|$ (respectively, $|C|$ and $|S|)$. Then any triple in $P$ is of the form $\left\{r_{i}, c_{j}, s_{k}\right\}$, and symbol $k$ can be thought of as occurring in row $i$ and column $j$ of an $n \times n$ array. Thus a partial latin square has at most one occurence of each symbol in any row or column, and each cell of the array has at most one symbol assigned to it. (If we replace 'at most one' in the preceeding sentence with 'exactly one' we have a latin square.)

A latin bitrade is a pair $(W, B)$ of nonempty partial latin squares such that for each triple $\left\{r_{i}, c_{j}, s_{k}\right\} \in W$ (respectively $B$ ) there exist unique $r_{i^{\prime}} \neq r_{i}$, $c_{j^{\prime}} \neq c_{j}$ and $s_{k^{\prime}} \neq s_{k}$ such that

$$
\left\{\left\{r_{i^{\prime}}, c_{j}, s_{k}\right\},\left\{r_{i}, c_{j^{\prime}}, s_{k}\right\},\left\{r_{i}, c_{j}, s_{k^{\prime}}\right\}\right\} \subset B(\text { respectively } W) .
$$

Each of $W$ and $B$ is called a latin trade, and we say that $W$ is the mate of $B$ (and vice versa). The size of the latin bitrade is $|W|$ (or $|B|$ ). It is possible for some trade, $W$ say, to occur in many distinct bitrades. 
Although latin trades have been studied implicitly in many different papers (generally when considering the difference between or the intersection of two latin squares of the same order) the first explicit appearance of latin trades in the literature is in a paper by Drápal and Kepka [14], where they are referred to as exchangeable partial groupoids. Subsequently to this they have been extensively studied (see [5] for a survey of results up until 2008).

In this paper we are motivated by the topological properties of latin trades. Let $\mathcal{G}$ be a 2 -cell embedding of a graph $G$ in a surface $\mathcal{S}$. If every face of $G$ is a 3 -cycle, then we say that $\mathcal{G}$ is a triangulation of $\mathcal{S}$. Now, consider a face 2-coloured triangulation of some surface, then each colour class corresponds to a partial triple system. The two partial triple systems $W$ and $B$ are said to be biembedded in the surface. If the triple systems are disjoint partial latin squares, then $(W, B)$ forms a latin bitrade, and we say that there is a biembedding of the bitrade $(W, B)$ into $\mathcal{S}$. In particular when the biembedding is in the sphere we say that $(W, B)$ is a spherical latin bitrade. We say that a latin bitrade is decomposable if there exist proper subsets $W^{\prime} \subseteq W$ and $B^{\prime} \subseteq B$ such that $\left(W^{\prime}, B^{\prime}\right)$ is a bitrade. If $(W, B)$ is an indecomposable latin bitrade of size $t$, then $|R|+|C|+|S| \leq t+2$, with equality exactly when $(W, B)$ is spherical. Thus the property of being spherical can be motivated purely combinatorially. In recent years biembeddings of bitrades have been extensively studied, see [9], 4], [10], [11] and [6].

Two partial latin squares are said to be isotopic if they are equal up to relabelling of their sets of rows, columns and symbols. A partial latin square $P$ is said to embed in an abelian group $A$ if there exist injective maps $f_{1}: R \rightarrow A, f_{2}: C \rightarrow A$ and $f_{3}: S \rightarrow A$ such that $f_{1}(r)+f_{2}(c)=f_{3}(s)$ for all $\{r, c, s\} \in P$. In other words, $P$ embeds in an abelian group $A$ if and only if it is isotopic to a partial latin subsquare $P^{\prime}$ of the multiplication table of $A$. (Of course, this definition can be easily generalised to nonabelian groups, indeed to general quasigroups.)

Lemma 1. Let $V=R \cup C \cup S$, where the sets $R, C$ and $S$ are disjoint. A partial latin square $P$ embeds in an abelian group $A$ if and only if there exists a function $f: V \rightarrow A$ that is injective when restricted to each of $R, C$ and $S$ and is such that $f(r)+f(c)+f(s)=0$ for all $\{r, c, s\} \in P$.

Proof. Using the notation in the definition of an embedding above, define $f$ by $\left.f\right|_{R}=f_{1},\left.f\right|_{C}=f_{2}$ and $\left.f\right|_{S}=-f_{3}$.

From now on, we refer to a function $f$ satisfying the conditions in Lemma 1 
as an embedding of $P$ in $A$. If the image of $f$ does not generate $A$, clearly $P$ may be embedded in a subgroup of $A$. We say that an abelian group $A$ is a minimal abelian representation for the partial latin square $P$ if $P$ embeds in $A$, and the image of $f$ generates $A$ for all embeddings $f$ of $P$ in $A$.

Note that the notion of an embedding of a partial transveral design $P$ in an abelian group only makes sense when the design is in fact a partial latin square, since the definition of an embedding implies that a triple in $P$ is determined uniquely by any two of its components.

In [7] Cavenagh and Drápal asked: "Can the individual trades in any biembedding of a latin bitrade be embedded into the operation table of an abelian group? If this is not true in general is it true for spherical latin bitrades?" This question for spherical latin bitrades was resolved positively by Canvenagh and Wanless in [6] (see also Drápal, Hämäläinen and Kala [12]), who also gave examples of biembeddings of latin bitrades $(W, B)$ into surfaces of higher genus such that $W$ does not embed into any group. Their work motivated their conjecture that $\mathcal{A}_{W} \cong \mathcal{A}_{B}$ for spherical latin bitrades $(W, B)$, where $\mathcal{A}_{W}$ and $\mathcal{A}_{B}$ are as defined in the introduction. Note that the definition of the group $\mathcal{A}_{W}$ makes sense for any partial transversal design $W$. However, the group $\mathcal{A}_{W}$ is particularly relevant once we know that $W$ is a partial latin square that embeds in some abelian group, because of the 'universal' property of $\mathcal{A}_{W}$ for embeddings of $W$, given by the following theorem. (A more general statement, for both abelian and nonabelian groups, was proved in [13].)

Theorem 2. Let $W$ be a partial latin square, and suppose there exists an embedding $f: V \rightarrow A$, where $A$ is an abelian group. Then the natural map $g: V \rightarrow \mathcal{A}_{W}$ is an embedding of $W$ in $\mathcal{A}_{W}$, and there is a homomorphism $h: \mathcal{A}_{W} \rightarrow A$ such that $f=h g$.

Proof. Let $F(V)$ be the free abelian group on the set $V$. There is a unique homomorphism $\zeta: F(V) \rightarrow A$ such that $\left.\zeta\right|_{V}=f$. The elements $r+c+s$ for $\{r, c, s\} \in W$ lie in the kernel of $\zeta$, since $f$ is an embedding of $W$ into $A$. Hence $\zeta$ induces a homomorphism $h: \mathcal{A}_{W} \rightarrow A$, and it is easy to see that $f=h g$. This implies that $g$ is injective when restricted to $R, C$ or $S$, and so $g$ is an embedding, as required.

In particular, Theorem 2 shows that any minimal abelian representation of $W$ is a quotient of $\mathcal{A}_{W}$. In fact, more is true. It is not hard to see that there is a homomorphism $\nu: \mathcal{A}_{W} \rightarrow \mathbb{Z} \oplus \mathbb{Z}$ such that $\nu(r)=(1,0), \nu(c)=(0,1)$ 
and $\nu(s)=(-1,-1)$ for $r \in R, c \in C$ and $s \in S$. Defining $\mathcal{C}_{W}$ to be the kernel of $\nu$, we see that $\mathcal{A}_{W} \cong \mathbb{Z} \oplus \mathbb{Z} \oplus \mathcal{C}_{W}$. (Here we are using the facts that $\nu$ is surjective and $\mathbb{Z} \oplus \mathbb{Z}$ is free abelian; see Rotman [18, Corollary 10.21], for example.) Both Drápal et al [12] and Cavenagh and Wanless [6] show that $\mathcal{C}_{W}$ is finite when $(W, B)$ is a spherical bitrade, so $\mathcal{C}_{W}$ is the torsion subgroup of $\mathcal{A}_{W}$. (They consider a group that is isomorphic to $\mathcal{C}_{W}$, which they call $\mathcal{A}_{W}^{*}$.) We will reprove this result in Section 5 .

It is worth noting as an aside that Cavenagh and Wanless have retracted their proof of the statement [6. Theorem 7] that the torsion subgroups of $\mathcal{A}_{W}$ and $\mathcal{A}_{B}$ have equal order. (Their proof of their Theorem 7 assumes, in their notation, that pairing each white face $f$ with the black face not adjacant to the vertex $v_{f}$ produces a bijection between sets of black and white faces. However, there are simple examples where this is not the case.) Of course, their Theorem 7 still holds, since it is a corollary of our Theorem 1 .

The following theorem is not stated explicitly by either Cavenagh and Wanless or Drápal et al, but can easily be proved from their work. (Although the result can also be obtained as a direct corollary of earlier work by Drápal and Kepka, Lemma 3.3 in [13], we believe that the proof we give is more accessible.)

Theorem 3. Let $W$ be a partial latin square that can be embedded in an abelian group. Define the group $\mathcal{C}_{W}$ as above. Then any minimal abelian representation $A$ of $W$ is a quotient of $\mathcal{C}_{W}$.

Proof. Let $f: V \rightarrow A$ be an embedding of $W$ in $A$, and let $g: V \rightarrow \mathcal{A}_{W}$ and $h: \mathcal{A}_{W} \rightarrow A$ be defined as in Theorem 2. Now $f(V)$ generates $A$, since $A$ is a minimal abelian representation of $W$. Since $h$ is a homomorphism and $h(g(V))=f(V)$, we see that $h$ is onto and so (by the first isomorphism theorem for groups) $A \cong Q$, where $Q=\mathcal{A}_{W} / K$ for the subgroup $K:=$ ker $h$ of $\mathcal{A}_{W}$. Without loss of generality, we may identify $A$ with $Q$.

Let $\bar{\nu}: Q \rightarrow(\mathbb{Z} \oplus \mathbb{Z}) / \nu(K)$ be the homomorphism induced from the map $\nu$ defined above. Let $r^{\prime} \in R, c^{\prime} \in C$ and $s^{\prime} \in S$ be fixed. Then $\bar{\nu}(f(R))=(1,0)+\nu(K)=\bar{\nu}\left(f\left(r^{\prime}\right)\right)$, and similarly $\bar{\nu}(f(C))=\bar{\nu}\left(f\left(c^{\prime}\right)\right)$ and $\bar{\nu}(S)=\bar{\nu}\left(f\left(s^{\prime}\right)\right)$. Define a new embedding $f^{\prime}$ of $W$ into $Q$ by

$$
f^{\prime}(v)=\left\{\begin{array}{l}
f(v)-f\left(r^{\prime}\right) \text { if } v \in R \\
f(v)-f\left(c^{\prime}\right) \text { if } v \in C \\
f(v)-f\left(s^{\prime}\right) \text { if } v \in S
\end{array}\right.
$$


Then $f^{\prime}$ is an embedding of $W$ in $Q$, and $\bar{\nu}\left(f^{\prime}(V)\right)=0$. But then $f^{\prime}(V) \subseteq$ ker $\bar{\nu}$ and so, since $Q$ is a minimal abelian representation of $W$, we must have $\operatorname{ker} \bar{\nu}=Q$. Thus $\bar{\nu}$ is the all zero map, and so $\nu(K)=\operatorname{im} \nu$. In particular, there exist $k_{1}, k_{2} \in K$ such that $\nu\left(k_{1}\right)=(1,0)$ and $\nu\left(k_{2}\right)=(0,1)$. We have that $\mathcal{A}_{W}=\left\langle k_{1}\right\rangle \oplus\left\langle k_{2}\right\rangle \oplus \mathcal{C}_{W}$. Setting $N=K \cap \mathcal{C}_{W}$, we find that $K=\left\langle k_{1}, k_{2}, N\right\rangle$ and so

$$
Q=\frac{\left\langle k_{1}\right\rangle \oplus\left\langle k_{2}\right\rangle \oplus \mathcal{C}_{W}}{\left\langle k_{1}, k_{2}, N\right\rangle} \cong \frac{\mathcal{C}_{W}}{N}
$$

\section{Connected bipartite planar graphs}

This section proves a key lemma that we need for the proof of Theorem 1 in Section 4.

Let $C$ and $S$ be non-empty finite sets. Let $\Gamma=(C \cup S, E)$ be a connected bipartite planar graph on the classes $C$ and $S$. Fix an embedding of $\Gamma$ in the sphere. Suppose there are $k$ faces $\mathcal{R}_{i}$ in this embedding. (When we apply the material in this section, $C$ and $S$ will be two of the colour classes of the vertex 3-colouring of our triangulation referred to in the introduction. If we write the third colour class as $R:=\left\{r_{1}, r_{2}, \ldots, r_{k}\right\}$, then $\mathcal{R}_{i}$ will be the region corresponding to the union of those triangles, whether black or white, containing $r_{i}$. So the boundary walk of $\mathcal{R}_{i}$ is the rotation at $r_{i}$.) For each face $\mathcal{R}_{i}$, let $e_{i 1}, e_{i 2}, \ldots, e_{i \ell_{i}}$ be the edges in the boundary of $\mathcal{R}_{i}$, taken clockwise. Let $u \in C \cup S$. For a set $X$, we write $F(X)$ for the free abelian group on the set $X$. For an edge $z \in E$ with end-vertex set $\psi(z)=\{s, c\}$, we define $\tilde{z}=s+c \in F(C \cup S)$ and $\tilde{E}=\{\tilde{z}: z \in E\}$. This section proves the following lemma.

Lemma 2. Let $\Gamma$ be a connected bipartite planar graph as above. Then $F(C \cup S)=\langle u\rangle_{\tilde{E}} \oplus\langle\tilde{E}\rangle$. We have that $\langle u\rangle \cong \mathbb{Z}$. Moreover, the natural map from $F(E)$ to $\langle\tilde{E}\rangle$ induces the following isomorphism:

$$
\langle\tilde{E}\rangle \cong F(E) /\left\langle\sum_{a=1}^{\ell_{i}}(-1)^{a} e_{i a}: i \in\{1,2, \ldots, k\}\right\rangle .
$$

Proof. We claim that $F(C \cup S)$ is generated by $\{u\} \cup \tilde{E}$. Let $u^{\prime} \in C \cup S \backslash\{u\}$. Since $\Gamma$ is connected, there is a path $u^{\prime}=u_{0}, u_{1}, \ldots, u_{\ell}=u$ from $u^{\prime}$ to $u$ in 
$\Gamma$, where $z_{i} \in E$ and $\psi\left(z_{i}\right):=\left\{u_{i}, u_{i+1}\right\}$ for all $i$. But then

$$
u^{\prime}+(-1)^{\ell-1} u=\sum_{i=0}^{\ell-1}(-1)^{i} \tilde{z}_{i} \in\langle\tilde{E}\rangle .
$$

So $u^{\prime} \in\langle\{u\} \cup \tilde{E}\rangle$ for all $u^{\prime} \in C \cup S$, and our claim follows.

Define a homomorphism $\phi: F(C \cup S) \rightarrow \mathbb{Z}$ by $\phi(c)=1$ for all $c \in C$ and $\phi(s)=-1$ for all $s \in S$. Since $\Gamma$ is bipartite with respect to the classes $C$ and $S$, we have that $\phi(\tilde{E})=0$. But $\phi(u)= \pm 1 \neq 0$. Thus $\langle u\rangle \cong \mathbb{Z}$ and $F(C \cup S)=\langle u\rangle \oplus\langle\tilde{E}\rangle$.

It remains to establish the last statement of the lemma. Let $\pi: F(E) \rightarrow$ $\langle\tilde{E}\rangle$ be the natural homomorphism, where $\pi(x)=\tilde{x}$ for all $x \in E$. It suffices to show that

$$
\operatorname{ker} \pi=\left\langle\sum_{a=1}^{\ell_{i}}(-1)^{a} e_{i a}: i \in\{1,2, \ldots, k\}\right\rangle .
$$

Clearly $\sum_{a=1}^{\ell_{i}}(-1)^{a} \tilde{e}_{i a}=0$ (since the first and last vertex of the boundary agree, and since $\ell_{i}$ is even as $\Gamma$ is bipartite). Thus the right hand side of (1) is contained in the left hand side.

Let $g \in \operatorname{ker} \pi$. We have that $g=\sum_{x \in E} f(x) x$ for some function $f: E \rightarrow$ $\mathbb{Z}$. The function $f$ is an integral edge weighting of $\Gamma$. If we orient the edges of $\Gamma$ from $C$ to $S$, the condition that $g \in \operatorname{ker} \pi$ is equivalent to the condition that $f$ lies in the integral flow space of $\Gamma$ (the set of edge-weightings with zero net flow at each vertex). We claim that the integral flow space is generated by the set of simple cycles of $\Gamma$ (flows in which all weights are \pm 1 , and the set of edges with non-zero weights form a cycle). This is a standard result (see [2, Proposition 7.13], for example), but for the reader's convenience we establish it here. Take a spanning tree $S$ for $\Gamma$, and for each edge $x \in \Gamma \backslash S$, let $C_{x}$ be the unique simple cycle in $S \cup\{x\}$, oriented so that $x$ appears with weighting 1. Given a flow $f$, the flow $f-\sum_{x \in \Gamma \backslash S} f(x) C_{x}$ is zero outside $S$, and so (since $S$ contains no cycles) is identically zero. Thus the set of simple cycles of the form $C_{x}$ generate the flow space, as claimed.

Since $\Gamma$ is planar, any simple cycle $\kappa$ is the sum of the boundary flows of those faces lying inside $\kappa$. Thus the flows corresponding to boundaries of faces generate ker $\pi$. But the boundary of face $\mathcal{R}_{i}$ is the element $\sum_{a=1}^{\ell_{i}}(-1)^{a} e_{i a}$, and so the lemma follows. 


\section{Proof of Theorem 1}

Let $\mathcal{G}$ be a face 2-coloured finite triangulation of the sphere. Let $W$ be the set of white triangles and $B$ be the set of black triangles in this triangulation. As above, let $V$ be the set of vertices of $\mathcal{G}$, and write $V=R \cup C \cup S$ where $R, C$ and $S$ are the colour classes of a 3-colouring of $V$.

Let $R=\left\{r_{1}, r_{2}, \ldots, r_{k}\right\}$. Define a $k \times k$ integer matrix $T=\left(t_{i j}\right)$ as follows. For $i \neq j$, define $t_{i j}$ to be the number of edges $e$, where $\psi(e)=\{c, s\}$, such that $\left\{r_{j}, c, s\right\} \in W$ and $\left\{r_{i}, c, s\right\} \in B$. Define $t_{i i}=-\sum_{j \neq i} t_{i j}$. Writing $d_{i}$ for the number of black triangles containing $r_{i}$, note that $d_{i}+t_{i i}$ is exactly the number of edges $e$, where $\psi(e)=\{c, s\}$, that occur in both a triangle $\left\{r_{i}, c, s\right\} \in W$ and a triangle $\left\{r_{i}, c, s\right\} \in B$.

Define a second matrix $T^{\prime}=\left(t_{i j}^{\prime}\right)$ in a similar way, but with the roles of black and white triangles reversed; so when $i \neq j$ we define $t_{i j}^{\prime}$ to be the number of edges $e$, where $\psi(e)=\{c, s\}$, such that $\left\{r_{j}, c, s\right\} \in B$ and $\left\{r_{i}, c, s\right\} \in W$, and we define $t_{i i}^{\prime}:=-\sum_{j \neq i} t_{i j}^{\prime}$. Writing $d_{i}^{\prime}$ for the number of white triangles containing $r_{i}$, we find that $d_{i}^{\prime}+t_{i i}^{\prime}$ is the number of edges $e$, where $\psi(e)=\{c, s\}$, that occur in both a triangle $\left\{r_{i}, c, s\right\} \in B$ and a triangle $\left\{r_{i}, c, s\right\} \in W$.

Clearly $t_{i j}^{\prime}=t_{j i}$ for $i \neq j$, and also $d_{i}+t_{i i}=d_{i}^{\prime}+t_{i i}^{\prime}$. Since the colour of the triangles containing $r_{i}$ alternates as we rotate about $r_{i}$, we have that $d_{i}=d_{i}^{\prime}$ and hence $t_{i i}=t_{i i}^{\prime}$. Thus $t_{i j}=t_{j i}^{\prime}$ for all $i, j \in\{1,2, \ldots, k\}$ and so $T^{\prime}$ is the transpose of $T$. One consequence of this fact is that both the row and the column sums of $T$ are all zero.

Define $\mathcal{B}_{W}$ to be the abelian group generated by elements $x_{1}, x_{2}, \ldots, x_{k}$, with relations matrix $T$. So

$$
\left.\mathcal{B}_{W}=\left\langle x_{1}, \ldots, x_{k}\right| \sum_{j=1}^{k} t_{i j} x_{j}=0 \text { for } i \in\{1,2, \ldots, k\}\right\rangle .
$$

Similarly, define $\mathcal{B}_{B}$ to have relations matrix $T^{\prime}$ :

$$
\left.\mathcal{B}_{B}=\left\langle x_{1}, \ldots, x_{k}\right| \sum_{j=1}^{k} t_{i j}^{\prime} x_{j}=0 \text { for } i \in\{1,2, \ldots, k\}\right\rangle .
$$

Since the Smith Normal Form of a $k \times k$ matrix is the same as the Smith Normal Form of its transpose, $\mathcal{B}_{W} \cong \mathcal{B}_{B}$. So establishing the following lemma will suffice to prove Theorem 1: 
Lemma 3. We have that $\mathcal{A}_{W} \cong \mathbb{Z} \oplus \mathcal{B}_{W}$ and $\mathcal{A}_{B} \cong \mathbb{Z} \oplus \mathcal{B}_{B}$.

Proof. We prove that $\mathcal{A}_{W} \cong \mathbb{Z} \oplus \mathcal{B}_{W}$. The proof that $\mathcal{A}_{B} \cong \mathbb{Z} \oplus \mathcal{B}_{B}$ is exactly the same, with the roles of black and white triangles interchanged, so we will omit it.

For each $i \in\{1,2, \ldots, k\}$, choose $c_{i} \in C$ and $s_{i} \in S$ so that $\left\{r_{i}, c_{i}, s_{i}\right\} \in$ $W$. We may use the relations $r_{i}+c_{i}+s_{i}=0$ to eliminate the generators $r_{i}$ from the presentation of $\mathcal{A}_{W}$. Each relation of the form $r_{i}+c+s=0$, where $\left\{r_{i}, c, s\right\}$ is a white triangle, becomes $(c+s)-\left(c_{i}+s_{i}\right)=0$, and so we have

$$
\mathcal{A}_{W}=F(C \cup S) /\langle U\rangle
$$

where $U$ is the set of relations of the form $(c+s)-\left(c_{i}+s_{i}\right)=0$ for each white triangle $\left\{r_{i}, c, s\right\}$.

Define $E$ to be the set of edges in the triangulation $\mathcal{G}$ whose endpoints both lie in $C \cup S$. The edges $E$ form a bipartite graph $\Gamma$ on $C \cup S$ with vertex classes $C$ and $S$. Since the edges of $E$ form part of a triangulation of the sphere, $\Gamma$ is planar. Moreover, $\Gamma$ is connected. To see this, note that if two triangles $\Delta_{1}, \Delta_{2}$ in $\mathcal{G}$ share an edge, then the edges of $\Delta_{1}$ and $\Delta_{2}$ that lie in $E$ have at least one vertex in common. Since a sphere is connected, any two triangles are connected by a path consisting of edge-adjacent triangles. So given two edges $e_{1}$ and $e_{2}$, where $\psi\left(e_{1}\right)=\{c, s\}$ and $\psi\left(e_{2}\right)=\left\{c^{\prime}, s^{\prime}\right\}$, an edge-adjacent path from a triangle containing $\{c, s\}$ to a triangle containing $\left\{c^{\prime}, s^{\prime}\right\}$ induces a path from $e_{1}$ to $e_{2}$ entirely consisting of edges in $E$.

Recall from Section 3 the notation $\tilde{x}=c+s$ for an element in the free abelian group $F(C \cup S)$ corresponding to an edge $x \in E$, where $\psi(x):=\{c, s\}$. For $i \in\{1,2, \ldots, k\}$, let $x_{i}$ be an edge such that $\psi\left(x_{i}\right)=\left\{s_{i}, c_{i}\right\}$ (where $s_{i}$ and $c_{i}$ were chosen above) adjacent to a white triangle $\left\{r_{i}, s_{i}, c_{i}\right\} \in W$. Note that the relations in $U$ are exactly those of the form $\tilde{x}-\tilde{x}_{i}=0$ where there is a white triangle containing $r_{i}$ and the edge $x$. Thus, in $\mathcal{A}_{W}$, every $\tilde{x} \in \tilde{E}$ is equal to $\tilde{x}_{i}$ for some $i$. Let $u \in C \cup S$. Since all the relations in $U$ lie in $\langle\tilde{E}\rangle$, Lemma 2 implies (in the notation there) that

$$
\begin{aligned}
\mathcal{A}_{W} & \cong\langle u\rangle \oplus(\langle\tilde{E}\rangle /\langle U\rangle) \\
& \cong \mathbb{Z} \oplus\left(F(E) /\left\langle U^{\prime} \cup\left\{\sum_{a=1}^{\ell_{i}}(-1)^{a} e_{i a}: i \in\{1,2, \ldots, k\}\right\}\right\rangle\right),
\end{aligned}
$$

where $U^{\prime}$ is a set of relations of the form $x-x_{i}=0$ for all $x \in E$ contained in a white triangle involving $r_{i}$. Recall that the boundary of region $\mathcal{R}_{i}$ consists 
of the edges $e_{i 1}, e_{i 2}, \ldots, e_{i \ell_{i}}$, in other words, the rotation at $r_{i}$ in $\mathcal{G}$. Thus the edges in the boundary are all contained in either a black or a white triangle that is adjacent to $r_{i}$, and the colour alternates as we travel round the boundary. We may choose to begin the boundary walk at an edge $e_{i 1}$ that is contained in a white triangle that is adjacent to $r_{i}$.

Each relation of the form $x-x_{i}=0$ can be used to eliminate one generator $x$ from our presentation of $\mathcal{A}_{W}$. Using all relations of this form, we can eliminate all generators other than $x_{1}, x_{2}, \ldots, x_{k}$ from our presentation. We claim that each relation of the form $\sum_{j=1}^{\ell_{i}}(-1)^{j} e_{i j}=0$ is transformed to the relation $\sum_{j=1}^{k} t_{i j} x_{j}=0$ during this elimination process. To see this, note that the edges $e_{i a}$ where $a$ is odd are exactly the edges of each of the $d_{i}^{\prime}$ white triangles containing $r_{i}$ and so are replaced by $x_{i}$. Each such edge appears with a negative sign in the relation, so $\sum_{a \text { odd }}(-1)^{a} e_{i a}=-d_{i}^{\prime} x_{i}$. The remaining edges lie in black triangles containing $r_{i}$, and so are replaced by various generators $x_{j_{a}}$ where $j_{a} \in\{1,2, \ldots, k\}$. When $i \neq j$, the definition of the integers $t_{i j}$ implies that exactly $t_{i j}$ of the edges are replaced by $x_{j}$. Moreover, we remarked when we defined the matrix $T$ that the number of edges $e$, where $\psi(e)=\{c, s\}$, that are contained in both a triangle $\left\{r_{i}, c, s\right\} \in B$ and a triangle $\left\{r_{i}, c, s\right\} \in W$ is exactly $d_{i}+t_{i i}$, where $d_{i}$ is the number of black triangles containing $r_{i}$. These $d_{i}+t_{i i}$ edges are replaced by $x_{i}$, and so

$$
\begin{aligned}
\sum_{a=1}^{\ell_{i}}(-1)^{a} e_{i a} & =-d_{i}^{\prime} x_{i}+\sum_{a \text { even }} e_{i a} \\
& =-d_{i}^{\prime} x_{i}+\left(d_{i}+t_{i i}\right) x_{i}+\sum_{j \neq i} t_{i j} x_{j} \\
& =\sum_{j=1}^{k} t_{i j} x_{j},
\end{aligned}
$$

since $d_{i}=d_{i}^{\prime}$. Thus our claim follows.

This change of presentation, together with (2) implies that

$$
\begin{aligned}
\mathcal{A}_{W} & \cong \mathbb{Z} \oplus\left(F\left(\left\{x_{1}, x_{2}, \ldots, x_{k}\right\}\right) /\left\langle\sum_{j=1}^{k} t_{i j} x_{j}: i \in\{1,2, \ldots, k\}\right\rangle\right) \\
& \cong \mathbb{Z} \oplus \mathcal{B}_{W},
\end{aligned}
$$

and so the lemma is proved. 


\section{The order of the torsion subgroup}

The matrix $T$ has determinant zero, since the entries in each row (and each column) sum to zero. Thus $\mathcal{B}_{W}$ is an infinite group. Cavenagh and Wanless show (in our notation) that $\mathcal{B}_{W} \cong \mathbb{Z} \oplus \mathcal{C}_{W}$, where $\mathcal{C}_{W}$ is finite. There is an alternative proof of this fact via Tutte's Matrix Tree Theorem (see Tutte [19, Chapter VI]), which we now give; this alternative proof has the advantage that it relates the order of $\mathcal{C}_{W}$ to the tree number of a certain directed graph $D$, which we now define.

Let $D$ be a directed graph on vertices $z_{1}, z_{2}, \ldots, z_{k}$, where we add exactly $t_{i j}$ directed edges from $z_{j}$ to $z_{i}$. So $D$ is a directed multigraph with no loops. In terms of our triangulation $\mathcal{G}$, the vertices of $D$ correspond to the regions $\mathcal{R}_{i}$ defined in Section 3 , with a directed edge from $z_{j}$ to $z_{i}$ for every white triangle in $\mathcal{R}_{j}$ that shares an edge with a black triangle in $\mathcal{R}_{i}$. In Tutte's terminology, $-T$ is the Kirchoff matrix of the directed graph $D$ with unit conductances. (This matrix is also known as the asymmetric Laplacian of $D$ : see Biggs [1].) Since the rows and columns of $T$ all sum to zero, we see that the in-degree and out-degree of any vertex $z_{i}$ of $D$ is zero; in other words, $D$ is Eulerian.

We claim that $D$ is strongly connected (in other words, for all ordered pairs of distinct vertices $z_{j}$ and $z_{i}$ there is an ordered path from $z_{j}$ to $z_{i}$ ). Let $j$ be fixed, and let $I \subseteq\{1,2, \ldots, k\}$ be defined by $i \in I$ if and only if there is a directed path from $z_{j}$ to $z_{i}$ in $D$. Clearly $j \in I$, so $I$ is non-empty. Let $\mathcal{S}:=\bigcup_{i \in I} \mathcal{R}_{i}$. Suppose, for a contradiction, that $I \neq\{1,2, \ldots, k\}$, so $\mathcal{S}$ is not the whole sphere. The border of $\mathcal{S}$ contains a non-trivial cycle consisting of edges whose end points lie in $C \cup S$. Since the edges with endpoints in $C \cup S$ form a bipartite graph, this cycle has at least two edges. Each edge of the cycle is adjacent to a unique triangle inside $\mathcal{S}$, and the colour of this triangle alternates as we move around the cycle. Thus there exists an edge $e$, where $\psi(e)=\{s, c\}$, on the border of $\mathcal{S}$ that is adjacent to a white triangle in $\mathcal{S}$ and a black triangle in $\mathcal{R}_{i^{\prime}}$ for some $i^{\prime} \notin I$. But then there is a directed edge in $D$ from some $z \in\left\{z_{i}: i \in I\right\}$ to $z_{i^{\prime}}$, and so $i^{\prime} \in I$. This contradiction shows that $I=\{1,2, \ldots, k\}$ and so $D$ is strongly connected as claimed.

Let $z$ be a vertex of $D$. An arborescence diverging from $z$ is a directed subtree of $D$ containing $z$ with all edges oriented away from $z$. The number $d$ of spanning arborescences in $D$ diverging from $z$ does not depend on $z$ (see [19, Theorem VI.23]); this number $d$ is known as the tree number $d$ of $D$. Since $D$ is strongly connected and Eulerian, $d>0$. Tutte shows [19, Theo- 
rem VI.28], as a corollary of the Matrix Tree Theorem, that $d=\operatorname{det}(-\bar{T})$, where $\bar{T}$ is the matrix obtained by removing the last row and column from $T$.

Theorem 4. Let $d$ be the (non-zero) tree number of the directed graph $D$ defined above. Then $\mathcal{B}_{W} \cong \mathbb{Z} \oplus \mathcal{C}_{W}$, where $\mathcal{C}_{W}$ is a finite abelian group of order $d$.

Proof. Since the row sums of the matrix $T$ defining $\mathcal{B}_{W}$ are all zero, the $\operatorname{map} \phi: \mathcal{B}_{W} \rightarrow \mathbb{Z}$ given by $\phi\left(\sum_{i=1}^{k} a_{i} x_{i}\right)=\sum_{i=1}^{k} a_{i}$ is well defined. Since $\phi\left(x_{k}\right)=1$, we see that $\phi$ is onto and $x_{k}$ has infinite order. Thus

$$
\mathcal{B}_{W}=\left\langle x_{k}\right\rangle \oplus \mathcal{C}_{W}
$$

where $\mathcal{C}_{W}=\operatorname{ker} \phi$ and $\left\langle x_{k}\right\rangle \cong \mathbb{Z}$.

Let $Q=\mathcal{B}_{W} /\left\langle x_{k}\right\rangle \cong \mathcal{C}_{W}$, and define $\bar{x}_{i}=x_{i}+\left\langle x_{i}\right\rangle \in Q$. Then $Q$ is generated by $\bar{x}_{1}, \bar{x}_{2}, \ldots, \bar{x}_{k-1}$, subject to the relations matrix $\bar{T}$ obtained from the matrix $T$ by removing its last row and column. We argued above that $\operatorname{det} \bar{T}=(-1)^{k-1} d \neq 0$, and so $\left|\mathcal{C}_{W}\right|=|Q|=d$, as required.

The structural insight provided by Theorem 4 enable us to construct examples of triangulations where the group $\mathcal{A}_{W}$ is large:

Theorem 5. Let $\mathcal{G}$ be the tiling of the sphere into $k$ regions $\mathcal{R}_{1}, \mathcal{R}_{2}, \ldots, \mathcal{R}_{k}$ given in Figure Q2. (In this tiling, $\mathcal{R}_{1}$ shares edges with $\mathcal{R}_{2}$ and $\mathcal{R}_{k}$. We identify vertices and edges appropriately; so for example $c_{k+1}=c_{1}$.) Then

$$
\mathcal{A}_{W} \cong \mathbb{Z} \oplus \mathbb{Z} \oplus \mathcal{C}_{W}
$$

where $\left|\mathcal{C}_{W}\right|=k 2^{k-1}$. Moreover, $\mathcal{A}_{W}$ has rank $k+1$.

Proof. Each region $\mathcal{R}_{i}$ contains exactly four black triangles; two share an edge with a white triangle in $\mathcal{R}_{i+1}$ and two with white triangles in $\mathcal{R}_{i-1}$ (subscripts being taken cyclically). Thus the relations matrix $T$ for $\mathcal{B}_{W}$ is circulant, with all entries on its main diagonal being -4 , bordered by two diagonals whose entries are all equal to 2 (and all other entries 0 ). The matrix $T$ induces a directed graph $D$, as defined before the statement of Theorem 4. (The graph $D$ is depicted in Figure 3 in the case when $k=6$.) Now, $D$ clearly has tree number $k 2^{k-1}$. The first statement of the theorem now follows from Theorem 4 , together with the fact that

$$
\mathcal{A}_{W} \cong \mathbb{Z} \oplus \mathcal{B}_{W} \cong \mathbb{Z} \oplus \mathbb{Z} \oplus \mathcal{C}_{W}
$$



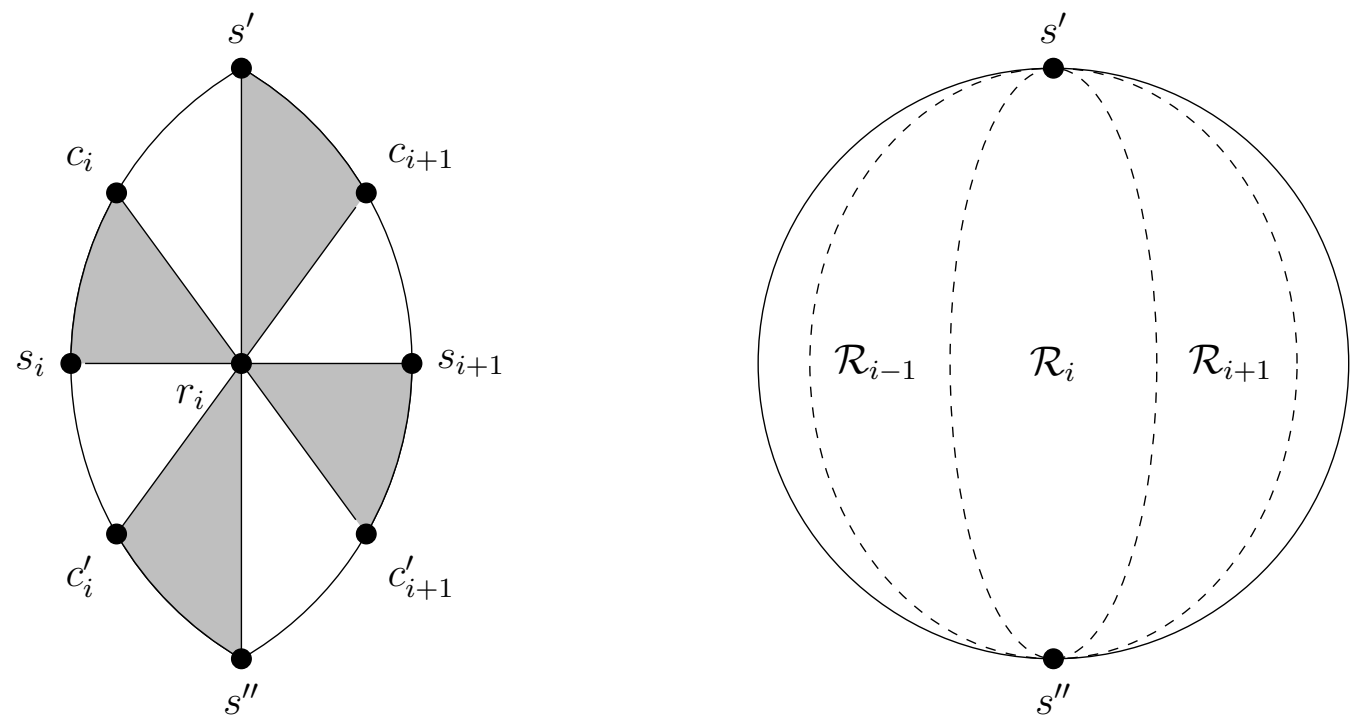

Figure 2: A typical region $\mathcal{R}_{i}$, and tiling the sphere with these regions.

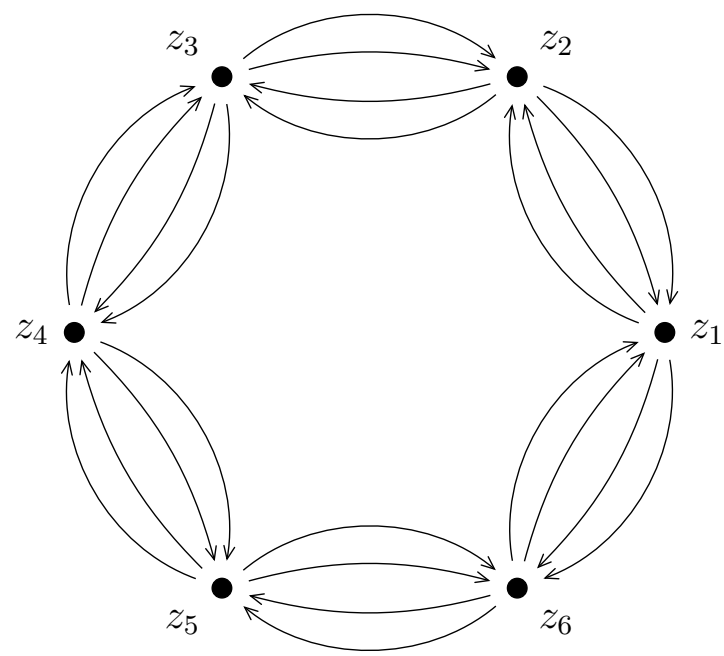

Figure 3: The directed graph $D$ when $k=6$. 
Since $\mathcal{B}_{W}$ is generated by $x_{1}, x_{2}, \ldots, x_{k}$, by definition, and since $\mathcal{A}_{W} \cong$ $\mathbb{Z} \oplus \mathcal{B}_{W}$, we see that the rank of $\mathcal{A}_{W}$ is at most $k+1$. So to prove the theorem, it suffices to show that the rank of $\mathcal{A}_{W}$ is at least $k+1$. In other words, we need to show that the rank of $\mathcal{B}_{W}$ is at least $k$.

Note that all the entries of the matrix $T$ are even, and so the relations given by the rows of $T$ are all consequences of relations $2 x_{i}=0$ for $i \in$ $\{1,2, \ldots, k\}$. So, if we write $[2] \mathcal{B}_{W}:=\left\{x+x: x \in \mathcal{B}_{W}\right\}$, we see that $\mathcal{B}_{W} /[2] \mathcal{B}_{W} \cong\left(\mathbb{Z}_{2}\right)^{k}$. But this quotient group has rank $k$, and so the rank of $\mathcal{B}_{W}$ is at least $k$, and the theorem follows.

We remark that there is no pair of disjoint edges $\left\{e_{1}, e_{2}\right\}$ with $\psi\left(e_{1}\right)=$ $\psi\left(e_{2}\right)$ in the triangulation in Theorem 5; nor are there distinct triangles sharing the same three vertices. So the triangulation in this example gives rise to a latin bitrade.

In the triangulation in Theorem [5, there are $t$ triangles of each colour, where $t=4 k$. So the order of the torsion subgroup $\mathcal{C}_{W}$ of $\mathcal{A}_{W}$ is exponential in $t$. Cavenagh and Wanless [6, Corollary 5] show that for any spherical latin bitrade with $t$ triangles of each colour, $\left|\mathcal{C}_{W}\right|<\frac{\sqrt{2}}{3} 6^{(t-1) / 3}$. The triangulation in Theorem 5 shows that an exponential upper bound on the order of the torsion subgroup of $\mathcal{A}_{W}$ is the best that could be hoped for (though it is quite possible that the constant in the exponential could be improved further).

We remarked in the proof of Theorem 5 that the rank of $\mathcal{A}_{W}$ is always bounded above by $k+1$. So the rank of $\mathcal{A}_{W}$ in Theorem 5 is maximal. Cavenagh and Wanless [6, Corollary 7] construct spherical latin bitrades where the rank of $\mathcal{A}_{W}$ grows at least logarithmically in $k$. Indeed, these bitrades have the extra property that whenever either partial latin square embeds in an abelian group $A$, the torsion rank of $A$ is bounded below by a logarithmic function of $k$. It would be interesting to know whether this logarithmic bound is close to being the best possible.

Finally, we remark that the construction in Theorem 5 can be easily generalised. Define a new triangulation, where each region $\mathcal{R}_{i}$ consists of $2 w$ black triangles, with $w$ of these triangles sharing an edge with white triangles in $\mathcal{R}_{i+1}$, and the remaining $w$ triangles sharing an edge with triangles in $\mathcal{R}_{i-1}$. (We tile the sphere with the regions $\mathcal{R}_{i}$ in the same way as in Theorem 5.) It is easy to show that for this triangulation $\left|\mathcal{C}_{W}\right|=k w^{k}$, and the group $\mathcal{B}_{W}$ has a quotient isomorphic to $\left(\mathbb{Z}_{w}\right)^{k}$. In particular, this triangulation shows that there is nothing special about the prime 2 here: for any prime $p$, there are examples of triangulations such that the $p$-primary subgroups of $\mathcal{C}_{W}$ have 
high rank.

\section{Comments}

This section is divided into two. The first subsection explores how far our results extend to triangulations of surfaces of higher genus. The second subsection states some questions and open problems regarding $\mathcal{A}_{W}$ for face 2-colourable triangulations of the sphere.

\subsection{Higher genus}

This section gives some examples that explore the extent to which Theorem 1 and Theorem 4 can be generalised.

The techniques in our paper depend crucially on the fact that the vertices of our triangulation can be vertex 3-coloured. For triangulations of non-spherical surfaces, this is no longer true in general, and so 2-colourable triangulations no longer necessarilly correspond to a biembedding of a pair of $\operatorname{PTD}_{\lambda}(3, n)$. Figure 4 gives three vertex non-3-colourable triangulations of the torus; these examples show that $\mathcal{A}_{W}$ no longer always has free rank 2 , and any straightforward generalisation of Theorem 4 is unlikely. Moreover, consider the (vertex non-3-colourable) nonorientable genus 3 triangulation on vertices $\{0,1,2,3,4,5,6,7,8\}$ with white and black triangles given by:

$$
\begin{aligned}
W & =\{012,034,057,068,135,146,178,236,247,258\} \\
B & =\{013,026,047,058,124,157,168,235,278,346\} .
\end{aligned}
$$

This example is due to Forbes, Grannell and Griggs [15. This triangulation has $\mathcal{A}_{W} \cong \mathbb{Z}_{3} \oplus \mathbb{Z}_{6}$ and $\mathcal{A}_{B} \cong \mathbb{Z}_{3} \oplus \mathbb{Z}_{3}$. Thus Theorem 1 cannot extend to this case. From Forbes et al, we can deduce that this triangulation is a counterexample with the smallest number of triangles if we restrict ourselves to having no pair of distinct edges $\left\{e_{1}, e_{2}\right\}$ with $\psi\left(e_{1}\right)=\psi\left(e_{2}\right)$ (in other words triangulations of simple graphs).

We now restrict ourselves to face 2-colourable triangulations that are also vertex 3-colourable. (Such triangulations give rise to biembeddings of $\left.\operatorname{PTD}_{\lambda}(3, n).\right)$ We note that the vertex colouring induces a consistent orientation on the faces of the triangulation, so the surfaces we consider are all orientable. 
(i)

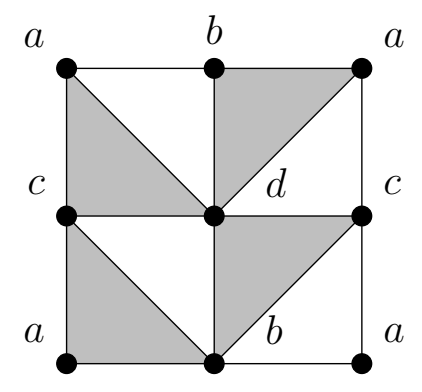

(ii)

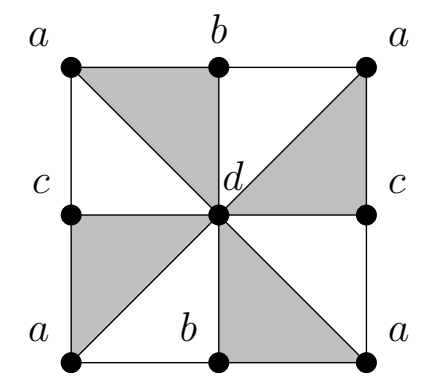

(iii)

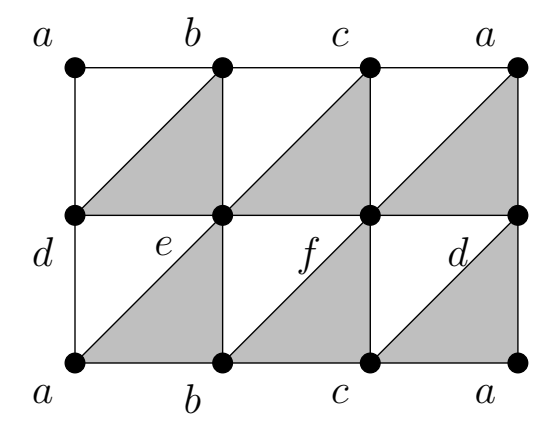

Figure 4: Three triangulations of the torus, with $\mathcal{A}_{W} \cong \mathbb{Z}_{3}, \mathcal{A}_{W} \cong \mathbb{Z} \oplus \mathbb{Z}$ and $\mathcal{A}_{W} \cong \mathbb{Z}_{9}$ respectively. 


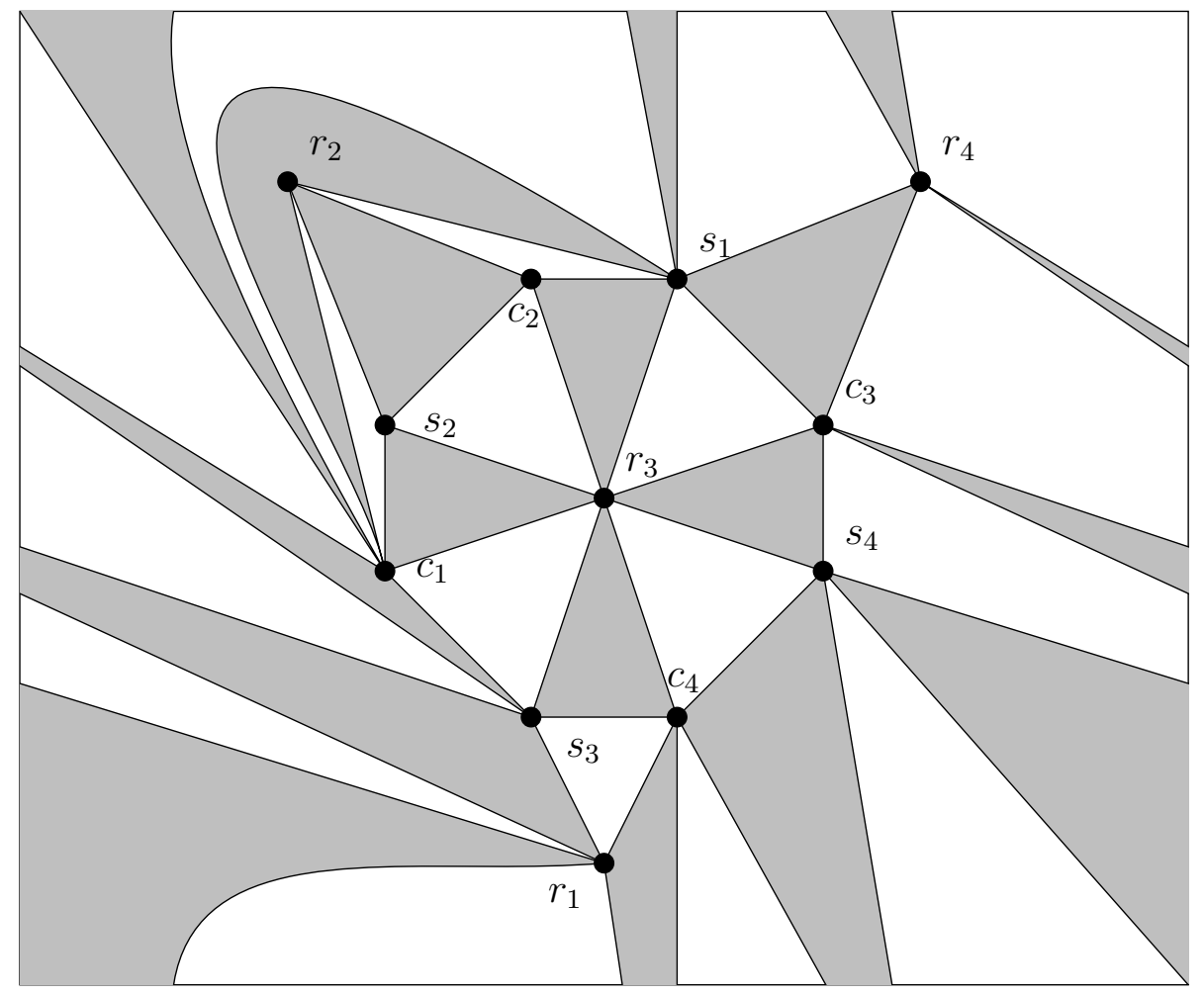

Figure 5: A face 2-coloured triangulation of the torus with $\mathcal{A}_{W} \cong \mathbb{Z} \oplus \mathbb{Z} \oplus \mathbb{Z}_{6}$ and $\mathcal{A}_{B} \cong \mathbb{Z} \oplus \mathbb{Z} \oplus \mathbb{Z}_{3}$.

Figure 5 gives an example of a vertex 3-colourable triangulation of the torus with $\mathcal{A}_{W} \cong \mathbb{Z} \oplus \mathbb{Z} \oplus \mathbb{Z}_{6}$ and $\mathcal{A}_{B} \cong \mathbb{Z} \oplus \mathbb{Z} \oplus \mathbb{Z}_{3}$. This example is due to Cavenagh and Wanless [6, Example 5.1]. Thus $\mathcal{A}_{W} ¥ \mathcal{A}_{B}$ in this example, and so Theorem 1 does not hold in general for vertex 3 -colourable surfaces of higher genus.

Nevertheless, the techniques of our paper do provide a weaker result in this more general setting. Let $\mathcal{G}$ be a vertex 3-coloured triangulation of some (orientable) surface, with faces 2-coloured black and white. Let $R, C$ and $S$ be the colour classes of the vertex 3-colouring. Define the groups $\mathcal{A}_{W}$, $\mathcal{A}_{B}, \mathcal{B}_{W}$ and $\mathcal{B}_{B}$ as before. Our analysis of the structure of $\mathcal{B}_{W}$ and $\mathcal{B}_{B}$ does not depend on the genus of the underlying surface (and so in particular, $\mathcal{B}_{W} \cong \mathcal{B}_{B} \cong \mathbb{Z} \oplus \mathcal{C}$, where $\mathcal{C}$ is finite). Indeed, the only time we used the fact that the underlying surface is a sphere was in Lemma 2 . For a graph $\Gamma$ 
embedded in an orientable surface of arbitrary genus, the statement of this lemma is still true if we replace the final isomorphism by the statement that $\langle\tilde{E}\rangle$ is a quotient of the group

$$
F(E) /\left\langle\sum_{a=1}^{\ell_{i}}(-1)^{a} e_{i a}: i \in\{1,2, \ldots, k\}\right\rangle .
$$

Thus we may argue, following the proofs of Theorems 11 and 4, that the following theorem is true.

Theorem 6. Let $\mathcal{G}$ be a face 2-coloured vertex 3-coloured triangulation of an orientable surface. Then $\mathcal{B}_{W} \cong \mathcal{B}_{B}$. Moreover, $\mathcal{A}_{W} \cong \mathbb{Z} \oplus \mathbb{Z} \oplus M$ and $\mathcal{A}_{B} \cong \mathbb{Z} \oplus \mathbb{Z} \oplus N$, where $M$ and $N$ are quotients of the (finite) torsion subgroup $\mathcal{C}_{W}$ of $\mathcal{B}_{W}$.

\subsection{Some open problems}

How large can the torsion subgroup $\mathcal{C}_{W}$ of $\mathcal{A}_{W}$ be? More precisely:

Question 1. Let $m_{t}$ be the maximal order of $\mathcal{C}_{W}$, over all 2-face colourable triangulations of the sphere with $t$ triangles of each colour. What is the value of $\lim \sup _{t \rightarrow \infty}\left(m_{t}\right)^{1 / t}$ ?

Theorem 5] and [6, Corollary 5] combine to show that this limit lies strictly between 1.189 and 1.818. In fact, the lower bound can be improved to 1.201 by using twelve rather than eight triangles in each region $\mathcal{R}_{i}$ (see the final paragraph of the previous section, with $w=3$ ). Question 1 asks whether this value can be determined precisely.

Both partial latin squares in a spherical latin bitrade $(W, B)$ embed in the finite group $\mathcal{C}_{W}$, but there is no reason to suppose that $\mathcal{C}_{W}$ is the group of minimal order with this property (though a minimal group must be isomorphic to a quotient of $\mathcal{C}_{W}$, by Theorem 3 ). Moreover, we believe that there should be examples of spherical latin bitrades $(W, B)$ such that $W$ and $B$ both embed in some abelian group of rank smaller than $\mathcal{C}_{W}$. Example 5.2 in Cavenagh and Wanless [6] is a spherical latin bitrade $(W, B)$ with $\mathcal{C}_{W} \cong \mathbb{Z}_{2} \oplus \mathbb{Z}_{6}$ (so has rank 2 ), where $W$ embeds in $\mathbb{Z}_{6}$ (of rank 1 ). But the smallest abelian group in which $B$ embeds in this example is $\mathcal{C}_{W}$ itself.

Question 2. Is there a family of bitrades with the property that the minimum order of an abelian group in which both mates embed is exponential in the size of the bitrade? 
Question 3. Is there a family of bitrades where the minimal rank of an abelian group in which both mates embed is linear in the size of the bitrade?

It is clear that the order of an abelian group in which both mates embed is at least linear in the size of the bitrade; [6. Corollary 7] shows that the minimal rank of an abelian group in which both mates embed can be at least logarithmic in the size of the bitrade.

\section{References}

[1] Norman Biggs, 'Algebraic potential theory on graphs', Bull. London Math. Soc. 29 (2007), 641-682.

[2] John A. Bondy and U. S. R. Murty, Graph Theory, Graduate Texts in Mathematics, 244. Springer, New York, 2008.

[3] Peter J. Cameron, 'Research problems from the BCC22', Discrete Math. 311 (2011), 1074-1083.

[4] Nicholas J. Cavenagh, 'Embedding 3-homogeneous latin trades into abelian 2-groups', Comentat. Math. Univ. Carolin. 45 (2004), 194-212.

[5] Nicholas J. Cavenagh, 'The theory and application of latin bitrades: a survey', Math. Slovaca 58 (2008), 691-718.

[6] Nicholas J. Cavenagh and Ian M. Wanless, 'Latin trades in groups defined on planar triangulations', J. Algebr. Comb. 30 (2009), 323-347.

[7] Aleš Drápal and Nick Cavenagh, Open Problem 8, 'Open problems from Workshop on latin trades', Prague, 6-10 February 2006. http://www.karlin.mff.cuni.cz/ rozendo/op.html

[8] Charles J. Colbourn and Jeffery H. Dinitz, (eds.), Second Edition, The CRC Handbook of Combinatorial Designs, Chapman \& Hall/CRC Press, Boca Raton (2006).

[9] Alěs Drápal, 'Hamming distances of groups and quasigroups', Discrete Math. 235 (2001) 189-197.

[10] Alěs Drápal, 'On elementary moves that generate all spherical Latin trades', Comment. Math. Univ. Carolin. 50 (2009), no. 4, 477-511. 
[11] Alěs Drápal, 'Geometrical structure and construction of Latin trades', Adv. Geom. 9 (2009), no. 3, 311-348.

[12] Alěs Drápal, Carlo Hämäläinen and Vítězslav Kala, 'Latin bitrades, dissections of equilateral triangles, and abelian groups', J. Comb. Des., 18 (2010), 1-24.

[13] Aleš Drápal and Tomáš Kepka, 'Group modifications of some partial groupoids', Annals of Discr. Math. 18 (1983), 319-332.

[14] Aleš Drápal and Tomáš Kepka, 'Exchangeable partial groupoids I', Acta Universitatis Carolinae - Mathematica et Physica 24 (1983), 57-72.

[15] Anthony D. Forbes, Mike J. Grannell and Terry S. Griggs, 'Configurations and trades in Steiner triple systems', Australasian J. Comb. 29 (2004), $75-84$.

[16] Percy J. Heawood, 'On the four-colour map theorem', Quart J. Pure Math. 29 (1898), 270-285.

[17] V.D. Mazurov and E.I. Khukhro (eds), The Kourovka notebook (Unsolved Problems in group theory), 17th edition, Russian Academy of Sciences, Siberian Division, Institute of Mathematics, Novosibirsk, 2010.

[18] Joseph J. Rotman, An Introduction to the Theory of Groups (3rd edition), Wm. C. Brown Publishers, Dubuque, Iowa, 1988.

[19] William T. Tutte, Graph theory, Addison-Wesley, Reading, MA, 1984.

[20] Robert A. Wilson, Graphs, colourings and the four-colour theorem, Oxford University Press, Oxford, 2002. 